\title{
Modelización espacial de ninfas de Bactericera cockerelli Sulc. en tomate de cáscara (Physalis ixocarpa Brot.) por medio de técnicas geoestadísticas
}

\author{
Spatial modeling of Bactericera cockerelli Sulc. nymphs on husk tomato (Physalis ixocarpa Brot.) using of \\ geostatistical techniques
}

\begin{abstract}
Roberto Rivera Martínez', José Francisco Ramírez Dávila²*, Marisol Martínez Quiroz², Andrés González Huerta² Universidad Autónoma del Estado de México. Toluca, México CP 50200.

2 Facultad de Ciencias Agrícolas, UAEM. Cerrillo Piedras Blancas s/n km 15 Carr. Toluca - Ixtlahuaca, entronque al Cerrillo. C.P. 50200; Toluca, Estado de México.
\end{abstract}

\section{RESUMEN}

El cultivo de tomate de cáscara es afectado por la presencia de diversas plagas y enfermedades en donde figura la presencia del psílido Bactericera cockerelli Sulc., al cual se le atribuye el amarillamiento y aborto floral. El control de este psílido no ha sido eficiente por el desconocimiento de la distribución espacial del mismo, por lo tanto, el presente estudio se realizó con la finalidad de conocer la distribución espacial de ninfas de Bactericera cockerelli, en tomate de cáscara por medio de técnicas geoestadísticas. Se determinó el semivariograma experimental y se ajustó a un modelo teórico, el ajuste se validó con el método de validación cruzada y se obtuvieron los mapas de agregación de la plaga a través del krigeado. Los resultados mostraron que las poblaciones de ninfas de Bactericera cockerelli, presentan una distribución del tipo agregada, la cual fue corroborada con los mapas de densidad. La plaga no infestó el $100 \%$ de la superficie de las parcelas estudiadas, lo cual nos ayuda a crear programas de manejo eficaces y dirigir las medidas de control a las áreas específicas de infestación.

Palabras Clave: Superficie infestada, Semivariogramas, Krigeado.

\section{ABSTRACT}

The husk tomato crop is affected by the presence of several pests and diseases which includes the presence of Bactericera cockerelli Sulc., psyllid to which, yellowing and floral abortion is attributed. The control of this psyllid has not been efficient; therefore, the present study was carriedout with the purpose to know the spatial distribution of $B$. cockerelli nymphs, on husk tomato by using geostatistical techniques. The experimental semivariogram was determined and a theoretical model adjusted, and validated with the cross validation method and the aggregation maps of the plate were obtained through krigeado. The results show that the populations of $B$. cockerelli nymphs exhibits an aggregate type distribution, corroborated with density maps. The pest did not infest $100 \%$ of the plots studied, which helps us to create effective management programs and direct control measures to specific areas of infestation.

Key Words: Infested surface, Semivariograms, Krigeado

\section{INTRODUCCIÓN}

El tomate de cáscara (Physalis ixocarpa Brot.) pertenece a la familia Solanaceae y subfamilia solanoideae tribu solaneae (contempla 18 géneros). Es una especie asociada a la vertiente del Pacífico, donde aún es posible hallársele en forma silvestre, en una franja que va desde Centroamérica (Guatemala), hasta California, E.U. (Pérez y Granados, 2001). La producción de tomate verde de cáscara representa el $4.25 \%$ de la superficie total sembrada de hortalizas a nivel nacional, teniendo un crecimiento promedio anual de $4.4 \%$. Alrededor del $81 \%$ del tomate producido en México es bajo condiciones de riego, el restante $19 \%$ es de temporal. El estado con mayor volumen de producción es Sinaloa, seguido de Nayarit, Michoacán, Jalisco, Sonora y el Estado de México. En el Estado de México la superficie cultivada es de 804 ha, con un volumen de producción en el ciclo otoño-invierno de $12,885 \mathrm{t}$ y un rendimiento de $16.01 \mathrm{t} \cdot \mathrm{ha}^{-1}$ (SIAP, 2017).

Uno de los principales problemas que enfrentan los productores de tomate son las patologías que se presentan cada ciclo, una de ellas se caracteriza por la presencia de plantas que envejecen antes de tiempo cuyos rendimientos disminuyen hasta un $60 \%$ comparado con el rendimiento esperado. En 2008, Liefting et al. determinaron que dichos síntomas están asociados con una nueva bacteria cuyo nombre propuesto es "Candidatus Liberibacter solanacearum" la cual parece ser la misma bacteria que causa el amarillamiento producido por los psílidos en tomate, de acuerdo con los investigadores referidos, esta bacteria es transmitida por el psílido B. cockerelli Sulc., que es un insecto ampliamente distribuido en la mayoría de las zonas agrícolas de México.

El monitoreo de las poblaciones de ninfas de B. cockerelli es un procedimiento muy importante en el manejo de este problema, ya que con ello se puede tener un panorama de cómo se encuentra distribuido dentro de las parcelas de estudio. Para poder utilizar adecuadamente la información derivada del monitoreo es necesario establecer puntos de muestreo que generen datos fehacientes con los cuales se podrá visualizar el comportamiento espacial de B. cockerelli. La infestación de este insecto-plaga, hace importante implementar actividades que permitan conocer su ubicación dentro de las parcelas de tomate de cascara;. Por lo tanto, el objetivo de este trabajo fue determinar el comportamiento 
espacial de las poblaciones de ninfas de Bactericera cockerelli Sulc., en el cultivo de tomate de cáscara (Physalis ixocarpa Brot.).

\section{MATERIALES Y MÉTODOS}

El estudio se llevó a cabo en los municipios de Luvianos, Ixtlahuaca y Jocotitlán, pertenecientes al Estado de México. Las parcelas estudiadas fueron establecidas utilizando sistema de riego, en las parcelas manejadas agronómicamente de forma similar y sin aplicaciones de agroquímicos. Los productores utilizaban el cultivar rendidora que se caracteriza por ser tener un desarrollo vigoroso de la planta alcanzando una altura promedio de $80 \mathrm{~cm}$ y sus ramas llegan a extenderse $1 \mathrm{~m}$ de longitud, la flor es grande de color amarillo con un diámetro de apertura de aproximadamente $2.5 \mathrm{~cm}$.

Para conocer el comportamiento espacial de B. cockerelli, se establecieron cuatro parcelas experimentales en cada uno de los municipios; en dichas parcelas se contabilizó el número de ninfas presentes en los tres estratos de la planta (baja, media, alta) tomando en cuenta cinco hojas de la planta. El muestreo utilizado fue por transectos, en el cual se estableció una malla rectangular de $100 \mathrm{~m}$ y se muestreo a cada $10 \mathrm{~m}$ en ambas direcciones, de este modo se muestrearon 121 plantas por parcela. Cada planta muestreada fue georreferenciada utilizando un DGPS marca Trimble modelo Pro XR, se cuantificó el número de ninfas por planta. Cabe señalar que en cada una de las parcelas experimentales se realizaron tres muestreos, el primer muestreo se realizó a los 30 días cuando las plantas se encontraban en floración, el segundo muestreo fue a los 40 días cuando iniciaba la fructificación y el último muestreo se realizó a los 60 días cuando la planta se encontraba en producción.

Se realizó una exploración estadística de los datos originales de las poblaciones de ninfas de $B$. cockerelli en los muestreos correspondientes a cada una de las parcelas. Utilizando la prueba de Curtosis se determinó que existía normalidad en los datos recolectados, por lo que no fue necesario realizar una transformación logarítmica de los datos $[\log 10(n+1)]$ para normalizarlos.

\section{Análisis geoestadístico}

Se obtuvo el semivariograma experimental a partir del valor promedio mensual de ninfas de $B$. cockerelli contadas en cada muestreo utilizando el programa Variowin 2.2 (Journel y Huijbregts, 1978; Isaaks y Srivastava, 1989). Una vez obtenido el semivariograma experimental, se realizó su ajuste a algún semivariograma teórico (esférico, exponencial, gaussiano, etc.) (Englund y Sparks, 1988) utilizando el programa Variowin versión 2.2. Una vez que el semivariograma se ajustó se sometió a un procedimiento de validación cruzada, en el cual los parámetros del modelo; efecto de pepita (Co), meseta $(C+C o)$ y rango o alcance (a) se van ajustando de forma interactiva (prueba y error) hasta obtener los mejores valores, este proceso usa los siguientes estadísticos: Media de los errores de estimación (MEE), Error cuadrático medio
(ECM) y Error cuadrático medio adimensional (ECMA).

La determinación del grado de relación entre los datos o nivel de dependencia espacial se obtuvo al dividir el efecto pepita entre la meseta y expresando en porcentaje su resultado. Si el resultado es menor de $25 \%$ el nivel de dependencia espacial es alta, si se encuentra entre 26 y $75 \%$ el nivel de dependencia espacial es moderado y si es mayor del $76 \%$ el nivel de dependencia es bajo (Cambardella et al., 1994).

Se realizó la interpolación de valores a través del krigeado ordinario que permite la estimación insesgada de valores asociados a puntos que no fueron muestreados, las estimaciones obtenidas fueron representadas en forma de mapa para cada fecha de muestreo mediante el uso del programa Surfer 9 (Surface Mapping System, 1993).

Es importante conocer si la infestación de la plaga era general en las parcelas de estudio. Para ello es importante conocer qué zonas están infestadas y el porcentaje que representan en el total de la parcela. Ello puede determinar el monto del ahorro económico y la aplicación de medidas de control. Su determinación se llevó a cabo mediante el software Surfer 9 (Surface Mapping System, 1993).

\section{RESULTADOS}

Previo al análisis geoestadístico se hizo un análisis estadístico dentro del cual se realizó una prueba de curtosis, para determinar si los datos recolectados provienen de una distribución normal. El coeficiente de curtósis indica normalidad cuando los valores están comprendidos en el rango de -3 a 3 ; en este caso, todos los valores estuvieron comprendidos dentro de este rango.

La distribución espacial que se obtuvo de poblaciones de $B$. cockerelli en los municipios estudiados fue de tipo agregada; los modelos a los que se ajustaron los semivariogramas fueron esféricos, gaussianos y exponenciales. En todos los muestreos y modelos ajustados se tuvo un efecto pepita igual a cero, dicho valor es indicativo de que la escala de muestreo utilizada fue la correcta y el error de muestreo es mínimo (Tabla 1).

Los valores del rango para las ninfas fueron de 7.2 y 52 $\mathrm{m}$ en el municipio de Luvianos, para Ixtlahuaca los valores se situaron entre 11.2 y $42.9 \mathrm{~m}$, mientras que para el municipio de Jocotitlán los valores se situaron entre 20.25 y $29.16 \mathrm{~m}$ (Tabla 1). El rango es la distancia máxima hasta la cual existe relación espacial entre los datos muestreados, ya que más allá del valor máximo de la distancia, la dependencia espacial es nula.

La media de población de ninfas de $B$. cockerelli en el municipio de Luvianos varió de 0.99 a 4.31 individuos, en Ixtlahuaca la densidad media fluctuó de 0.17 a 2.60 ninfas, mientras que para Jocotitlán el promedio se situó entre 0.17 a 15.44 ninfas por planta (Tabla 1).

Los resultados de la validación cruzada están concentrados en la Tabla 2, en el cual los valores de los estadísticos estuvieron dentro del rango que permitió la validación de los modelos ajustados. Todos los modelos presentaron un alto 
Tabla 1. Parámetros (efecto pepita, meseta y rango) de los modelos ajustados a los semivariogramas de Bactericera cockerelli Sulc.

Table 1. Parameters (nugget effect, plateau and range) of the models adjusted to the semivariograms of Bactericera cockerelli Sulc.

\begin{tabular}{|c|c|c|c|c|c|c|c|c|}
\hline & Muestreo & Modelo & $\begin{array}{l}\text { Densidad } \\
\text { Promedio }\end{array}$ & Pepita & Meseta & Rango & $\begin{array}{c}\text { Pepita/ } \\
\text { Meseta } \\
\%\end{array}$ & $\begin{array}{l}\text { Nivel de depen- } \\
\text { dencia espacial }\end{array}$ \\
\hline \multicolumn{9}{|l|}{ Luvianos } \\
\hline \multirow{3}{*}{ Parcela 1} & M1 & Gaussiano & 2.48 & 0 & 4.37 & 12.48 & 0 & Alta \\
\hline & M2 & Gaussiano & 1.15 & 0 & 4.37 & 12.48 & 0 & Alta \\
\hline & M3 & Esférico & 1.34 & 0 & 2.87 & 17.28 & 0 & Alta \\
\hline \multirow{3}{*}{ Parcela 2} & M1 & Esférico & 4.30 & 0 & 3.15 & 24.2 & 0 & Alta \\
\hline & M2 & Esférico & 0.99 & 0 & 3.39 & 8.8 & 0 & Alta \\
\hline & M3 & Gaussiano & 1.15 & 0 & 36.62 & 11 & 0 & Alta \\
\hline \multirow{3}{*}{ Parcela 3} & M1 & Exponencial & 1.19 & 0 & 12.39 & 52 & 0 & Alta \\
\hline & M2 & Esférico & 1.76 & 0 & 5.48 & 26 & 0 & Alta \\
\hline & M3 & Esférico & 3.47 & 0 & 3.29 & 27.2 & 0 & Alta \\
\hline \multirow{3}{*}{ Parcela 4} & M1 & Esférico & 1.60 & 0 & 18.18 & 7.2 & 0 & Alta \\
\hline & M2 & Esférico & 3.05 & 0 & 9.46 & 8.5 & 0 & Alta \\
\hline & M3 & Esférico & 4.31 & 0 & 5.18 & 11.2 & 0 & Alta \\
\hline \multicolumn{9}{|c|}{ Ixtlahuaca } \\
\hline \multirow{3}{*}{ Parcela 1} & M1 & Gaussiano & 0.20 & 0 & 0.24 & 16.1 & 0 & Alta \\
\hline & M2 & Esférico & 1.23 & 0 & 4.8 & 21.6 & 0 & Alta \\
\hline & M3 & Exponencial & 0.17 & 0 & 0.26 & 11.2 & 0 & Alta \\
\hline \multirow{3}{*}{ Parcela 2} & M1 & Esférico & 0.97 & 0 & 1.64 & 40.8 & 0 & Alta \\
\hline & M2 & Esférico & 1.11 & 0 & 1.77 & 38.4 & 0 & Alta \\
\hline & M3 & Exponencial & 2.60 & 0 & 5.57 & 26.27 & 0 & Alta \\
\hline \multirow{3}{*}{ Parcela 3} & M1 & Exponencial & 0.74 & 0 & 1.29 & 41.46 & 0 & Alta \\
\hline & M2 & Esférico & 0.57 & 0 & 1.13 & 42.9 & 0 & Alta \\
\hline & M3 & Esférico & 1.33 & 0 & 0.93 & 31.9 & 0 & Alta \\
\hline \multirow{3}{*}{ Parcela 4} & M1 & Exponencial & 0.55 & 0 & 1.43 & 12.48 & 0 & Alta \\
\hline & M2 & Esférico & 0.66 & 0 & 4.04 & 17.28 & 0 & Alta \\
\hline & M3 & Exponencial & 1.04 & 0 & 2.84 & 15.36 & 0 & Alta \\
\hline \multicolumn{9}{|c|}{ Jocotitlán } \\
\hline \multirow{3}{*}{ Parcela 1} & M1 & Gaussiano & 0.17 & 0 & 0.24 & 29.16 & 0 & Alta \\
\hline & M2 & Esférico & 1.63 & 0 & 3.86 & 20.25 & 0 & Alta \\
\hline & M3 & Esférico & 4.52 & 0 & 19.51 & 20.25 & 0 & Alta \\
\hline \multirow{3}{*}{ Parcela 2} & M1 & Esférico & 1.58 & 0 & 5.87 & 23.2 & 0 & Alta \\
\hline & M2 & Esférico & 0.96 & 0 & 3.3 & 28 & 0 & Alta \\
\hline & M3 & Esférico & 2.51 & 0 & 11.83 & 24.8 & 0 & Alta \\
\hline \multirow{3}{*}{ Parcela 3} & M1 & Esférico & 1.76 & 0 & 3.94 & 23.49 & 0 & Alta \\
\hline & M2 & Esférico & 1.05 & 0 & 3.25 & 21.87 & 0 & Alta \\
\hline & M3 & Esférico & 2.66 & 0 & 8.42 & 25.92 & 0 & Alta \\
\hline \multirow{3}{*}{ Parcela 4} & M1 & Esférico & 1.98 & 0 & 13.13 & 25.6 & 0 & Alta \\
\hline & M2 & Esférico & 1.92 & 0 & 13.12 & 21.6 & 0 & Alta \\
\hline & M3 & Esférico & 15.44 & 0 & 75.24 & 24 & 0 & Alta \\
\hline
\end{tabular}


Tabla 2. Valores de los estadísticos de la validación cruzada de los semivariogramas obtenidos en los muestreos de Bactericera cockerelli Sulc: media de los errores de estimación (MEE), error cuadrático medio (ECM) y error cuadrático medio adimensional (ECMA).

Table 2. Values of the cross-validation statistics of the semivariograms obtained in samplings of Bactericera cockerelli Sulc: mean estimation errors (MEE), mean square error (ECM) and mean square quadratic error (ECMA).

\begin{tabular}{|c|c|c|c|c|c|c|}
\hline & Muestreo & Varianza muestral & MEE* & $\begin{array}{c}\text { Varianza de los } \\
\text { errores }\end{array}$ & ECM & ECMA \\
\hline \multicolumn{7}{|l|}{ Luvianos } \\
\hline \multirow{3}{*}{ Parcela 1} & Muestreo 1 & 8.78 & $0.10^{\text {ns }}$ & 5.23 & 0.04 & 1.10 \\
\hline & Muestreo 2 & 4.70 & $0.11^{\text {ns }}$ & 1.27 & 0.09 & 1.11 \\
\hline & Muestreo 3 & 3.07 & $0.10^{\mathrm{ns}}$ & 1.04 & 0.10 & 1.14 \\
\hline \multirow{3}{*}{ Parcela 2} & Muestreo 1 & 39.99 & $0.12^{\mathrm{ns}}$ & 23.64 & 0.14 & 1.13 \\
\hline & Muestreo 2 & 3.82 & $0.11^{\mathrm{ns}}$ & 1.22 & 0.16 & 1.06 \\
\hline & Muestreo 3 & 3.95 & $0.13^{\text {ns }}$ & 2.17 & 0.11 & 1.07 \\
\hline \multirow{3}{*}{ Parcela 3} & Muestreo 1 & 3.41 & $0.11^{\mathrm{ns}}$ & 2.68 & 0.15 & 1.12 \\
\hline & Muestreo 2 & 6.21 & $0.12^{\mathrm{ns}}$ & 4.92 & 0.10 & 1.10 \\
\hline & Muestreo 3 & 13.51 & $0.10^{\mathrm{ns}}$ & 10.46 & 0.08 & 1.09 \\
\hline \multirow{3}{*}{ Parcela 4} & Muestreo 1 & 6.55 & $0.11^{\mathrm{ns}}$ & 5.25 & 0.11 & 1.13 \\
\hline & Muestreo 2 & 13.92 & $0.10^{\mathrm{ns}}$ & 11.74 & 0.13 & 1.15 \\
\hline & Muestreo 3 & 20.25 & $0.13^{\mathrm{ns}}$ & 17.61 & 0.14 & 1.05 \\
\hline \multicolumn{7}{|c|}{ Ixtlahuaca } \\
\hline \multirow{3}{*}{ Parcela 1} & Muestreo 1 & 0.34 & $0.12^{\mathrm{ns}}$ & 0.14 & 0.13 & 1.12 \\
\hline & Muestreo 2 & 8.01 & $0.11^{\mathrm{ns}}$ & 6.47 & 0.11 & 1.15 \\
\hline & Muestreo 3 & 0.26 & $0.10^{\mathrm{ns}}$ & 0.12 & 0.10 & 1.08 \\
\hline \multirow{3}{*}{ Parcela 2} & Muestreo 1 & 1.79 & $0.12^{\mathrm{ns}}$ & 1.08 & 0.18 & 1.04 \\
\hline & Muestreo 2 & 1.83 & $0.11^{\mathrm{ns}}$ & 1.15 & 0.19 & 1.11 \\
\hline & Muestreo 3 & 5.85 & $0.13^{\mathrm{ns}}$ & 3.96 & 0.08 & 1.14 \\
\hline \multirow{3}{*}{ Parcela 3} & Muestreo 1 & 2.75 & $0.12^{\mathrm{ns}}$ & 1.77 & 0.11 & 1.06 \\
\hline & Muestreo 2 & 1.34 & $0.10^{\mathrm{ns}}$ & 1.02 & 0.12 & 1.10 \\
\hline & Muestreo 3 & 1.02 & $0.11^{\mathrm{ns}}$ & 0.75 & 0.15 & 1.09 \\
\hline \multirow{3}{*}{ Parcela 4} & Muestreo 1 & 0.83 & $0.13^{\mathrm{ns}}$ & 0.44 & 0.10 & 1.12 \\
\hline & Muestreo 2 & 1.60 & $0.10^{\mathrm{ns}}$ & 1.23 & 0.12 & 1.07 \\
\hline & Muestreo 3 & 3.18 & $0.11^{\mathrm{ns}}$ & 2.14 & 0.06 & 1.05 \\
\hline \multicolumn{7}{|l|}{ Jocotitlán } \\
\hline \multirow{3}{*}{ Parcela 1} & Muestreo 1 & 0.32 & $0.10^{\mathrm{ns}}$ & 0.14 & 0.11 & 1.14 \\
\hline & Muestreo 2 & 4.81 & $0.12^{\mathrm{ns}}$ & 3.20 & 0.04 & 1.07 \\
\hline & Muestreo 3 & 21.46 & $0.13^{\mathrm{ns}}$ & 15.85 & 0.10 & 1.11 \\
\hline \multirow{3}{*}{ Parcela 2} & Muestreo 1 & 5.91 & $0.12^{\mathrm{ns}}$ & 4.05 & 0.11 & 1.09 \\
\hline & Muestreo 2 & 3.31 & $0.13^{\mathrm{ns}}$ & 2.17 & 0.01 & 1.10 \\
\hline & Muestreo 3 & 13.15 & $0.15^{\mathrm{ns}}$ & 10.82 & 0.09 & 1.13 \\
\hline \multirow{3}{*}{ Parcela 3} & Muestreo 1 & 4.21 & $0.10^{\mathrm{ns}}$ & 2.85 & 0.05 & 1.04 \\
\hline & Muestreo 2 & 3.37 & $0.12^{\mathrm{ns}}$ & 2.07 & 0.12 & 1.12 \\
\hline & Muestreo 3 & 10.52 & $0.14^{\mathrm{ns}}$ & 8.93 & 0.10 & 1.07 \\
\hline \multirow{3}{*}{ Parcela 4} & Muestreo 1 & 14.96 & $0.13^{\mathrm{ns}}$ & 11.39 & 0.09 & 1.13 \\
\hline & Muestreo 2 & 3.25 & $0.10^{\mathrm{ns}}$ & 2.17 & 0.13 & 1.10 \\
\hline & Muestreo 3 & 88.19 & $0.14^{\text {ns }}$ & 56.28 & 0.04 & 1.08 \\
\hline
\end{tabular}

ns: no significativo al 5\%; s: significativo al 5\% 
Tabla 3. Porcentajes de superficie infestada y no infestada obtenida en los muestreos de ninfas de Bactericera cockerelli Sulc., en los 3 municipios.

Table 3. Percentages of infested and non-infested surface obtained in the sampling of Bactericera cockerelli Sulc. nymphs, in the 3 municipalities.

\begin{tabular}{|c|c|c|c|}
\hline \multicolumn{4}{|c|}{ Luvianos } \\
\hline & Muestreo & $\%$ No Infestado & \% Infestación \\
\hline \multirow{3}{*}{ Parcela 1} & Muestreo 1 & 9 & 91 \\
\hline & Muestreo 2 & 7 & 93 \\
\hline & Muestreo 3 & 6 & 94 \\
\hline \multirow{3}{*}{ Parcela 2} & Muestreo 1 & 5 & 95 \\
\hline & Muestreo 2 & 7 & 93 \\
\hline & Muestreo 3 & 6 & 94 \\
\hline \multirow{3}{*}{ Parcela 3} & Muestreo 1 & 3 & 97 \\
\hline & Muestreo 2 & 7 & 93 \\
\hline & Muestreo 3 & 10 & 90 \\
\hline \multirow{3}{*}{ Parcela 4} & Muestreo 1 & 13 & 87 \\
\hline & Muestreo 2 & 11 & 89 \\
\hline & Muestreo 3 & 7 & 93 \\
\hline \multicolumn{4}{|c|}{ Ixtlahuaca } \\
\hline \multirow{3}{*}{ Parcela 1} & Muestreo 1 & 21 & 79 \\
\hline & Muestreo 2 & 23 & 77 \\
\hline & Muestreo 3 & 19 & 81 \\
\hline \multirow{3}{*}{ Parcela 2} & Muestreo 1 & 12 & 88 \\
\hline & Muestreo 2 & 14 & 86 \\
\hline & Muestreo 3 & 15 & 85 \\
\hline \multirow{3}{*}{ Parcela 3} & Muestreo 1 & 23 & 77 \\
\hline & Muestreo 2 & 20 & 80 \\
\hline & Muestreo 3 & 15 & 85 \\
\hline \multirow{3}{*}{ Parcela 4} & Muestreo 1 & 10 & 90 \\
\hline & Muestreo 2 & 15 & 85 \\
\hline & Muestreo 3 & 14 & 86 \\
\hline \multicolumn{4}{|c|}{ Jocotitlán } \\
\hline \multirow{3}{*}{ Parcela 1} & Muestreo 1 & 16 & 84 \\
\hline & Muestreo 2 & 13 & 87 \\
\hline & Muestreo 3 & 10 & 90 \\
\hline \multirow{3}{*}{ Parcela 2} & Muestreo 1 & 17 & 83 \\
\hline & Muestreo 2 & 15 & 85 \\
\hline & Muestreo 3 & 16 & 84 \\
\hline \multirow{3}{*}{ Parcela 3} & Muestreo 1 & 11 & 89 \\
\hline & Muestreo 2 & 15 & 85 \\
\hline & Muestreo 3 & 18 & 82 \\
\hline \multirow{3}{*}{ Parcela 4} & Muestreo 1 & 15 & 85 \\
\hline & Muestreo 2 & 11 & 89 \\
\hline & Muestreo 3 & 7 & 93 \\
\hline
\end{tabular}

\section{Volumen XXII, Número 1}


nivel de dependencia espacial para cada una de las fechas de muestreo en los tres municipios. Los modelos de la distribución espacial de ninfas de $B$. cockerelli en las parcelas estudiadas se validaron con los parámetros estadísticos al ubicarse dentro del rango permisible.

Los mapas de la superficie infestada para los municipios de Luvianos e Ixtlahuaca indicaron que los centros de agregación se encontraron distribuidos de forma general dentro de la parcela, sin embargo, los centros más marcados se situaron hacia los bordes de la parcela (color oscuro). En cuanto al municipio de Jocotitlán los centros de agregación se distribuyeron dentro de toda la parcela (Figura 1, 2 y 3)

En los tres municipios se observó que en todos los muestreos de las cuatro parcelas la infestación no alcanzo el $100 \%$, sin embargo si se presentaron infestaciones altas, ya que en Luvianos se obtuvo un $97 \%$ de infestación en la parcela 3; el menor porcentaje se obtuvo en la parcela 4 con un $87 \%$, para Ixtlahuaca la mayor infestación fue de $90 \%$ en
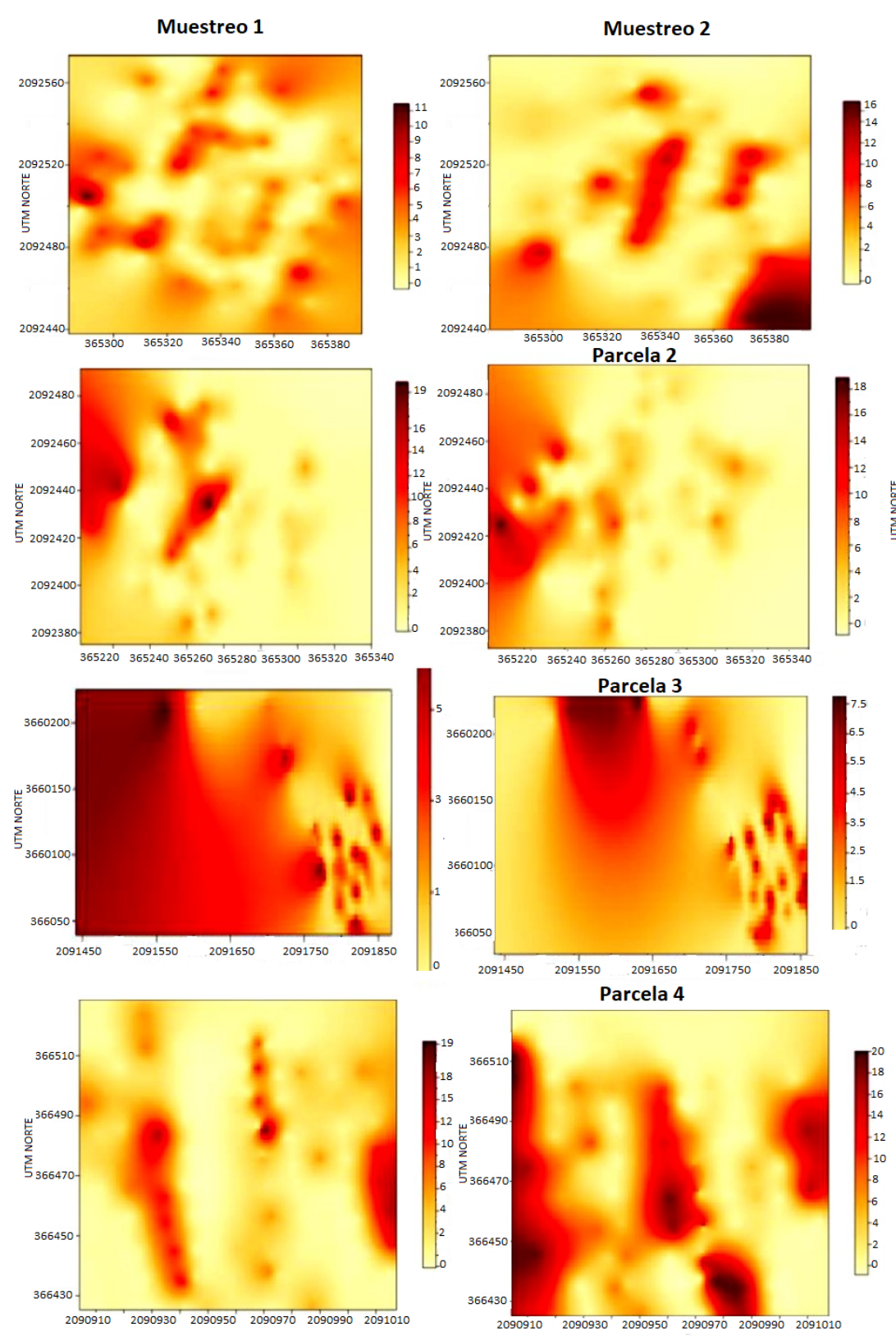
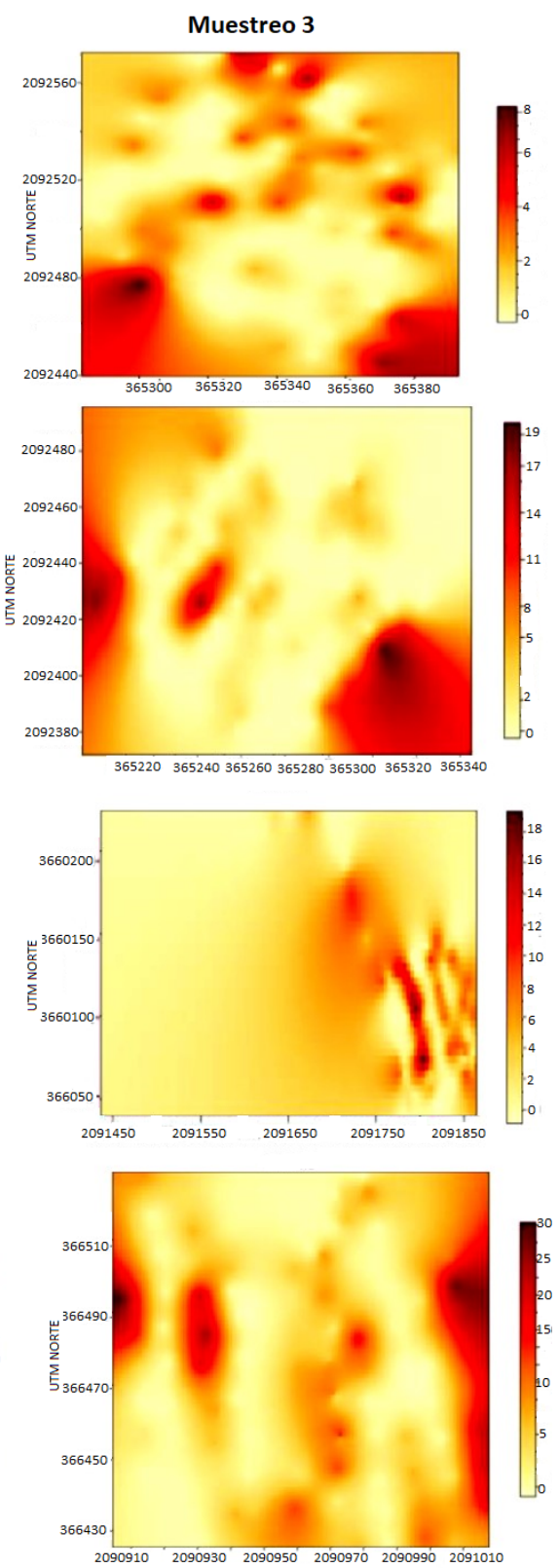

Figura 1. Mapas de densidad de las poblaciones de ninfas de Bactericera cockerelli Sulc., obtenidos en las parcelas del municipio de Luvianos Estado de México.

Figure 1. Nymph populations density maps of Bactericera cockerelli Sulc., obtained in the plots of the municipality of Luvianos State of Mexico. 
la parcela 4 y la menor en la parcela 1 y 3 con un 77\%, mientras que en Jocotitlán se obtuvo un $93 \%$ de infestación en la parcela 4 y un $82 \%$ en la parcela 3 . Estos porcentajes indican que la plaga no se estableció en el $100 \%$ de la parcela, lo cual resulta interesante a la hora de realizar medidas de control, ya que esta información será de utilidad para aplicar control focalizado en áreas de infestación.

Los resultados obtenidos en este estudio se pueden presentar ante las autoridades competentes para que se pue- dan desarrollar programas de manejo integrado de la plaga a largo plazo, ya que, con este tipo de tecnologías aplicadas a la agricultura, es posible dirigir las medidas de control (sean químicas, biológicas, físicas, mecánicas o culturales) a sitios específicos donde se ubica la plaga, esto debido a que se conoce el comportamiento espacial de la misma, y de esta manera se pueden tener ahorros económicos y menor impacto ambiental al dejar de hacer aplicaciones generales dentro del cultivo.
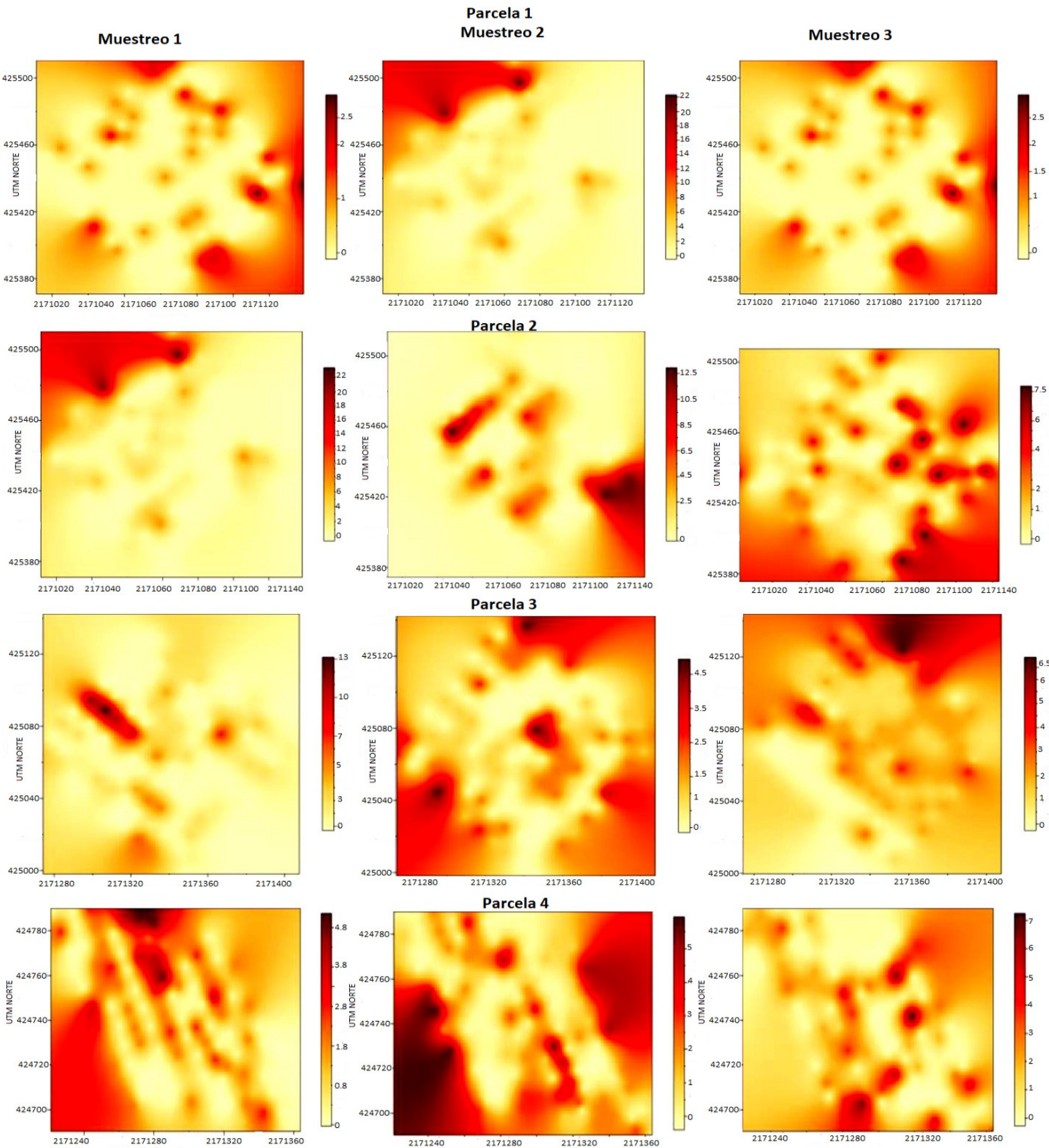

Figura 2. Mapas de densidad de las poblaciones de ninfas de Bactericera cockerelli Sulc., obtenidos en las parcelas del municipio de Ixtlahuaca Estado de México.

Figure 2. Nymph populations density maps of Bactericera cockerelli Sulc., obtained in the plots of the municipality of Ixtlahuaca, State of Mexico. 


\section{DISCUSIÓN}

La observación de la estructura agregada en la distribución espacial de ninfas de Bactericera cockerelli en el cultivo de tomate de cáscara fue posible realizarse con el uso de la geoestadística. Samper y Carrera, 1996 mencionan que el estudio de la distribución de un organismo utilizando los métodos geoestadísticos tienen ventaja sobre los métodos donde se utiliza la estadística clásica, ya que la geoestadística toma en cuenta datos georreferenciados con lo que se toma en cuenta su localización espacial.

La geoestadística además permite elaborar mapas de densidad de la distribución espacial de un organismo (Rossi et al., 1992; Ribes et al., 1998; Ramírez et al., 2011; Maldonado et al., 2017). Acosta et al. (2017) utilizaron la geoestadística para estudiar la distribución espacial de trips en aguacate, además obtuvieron mapas de densidad para los muestreos
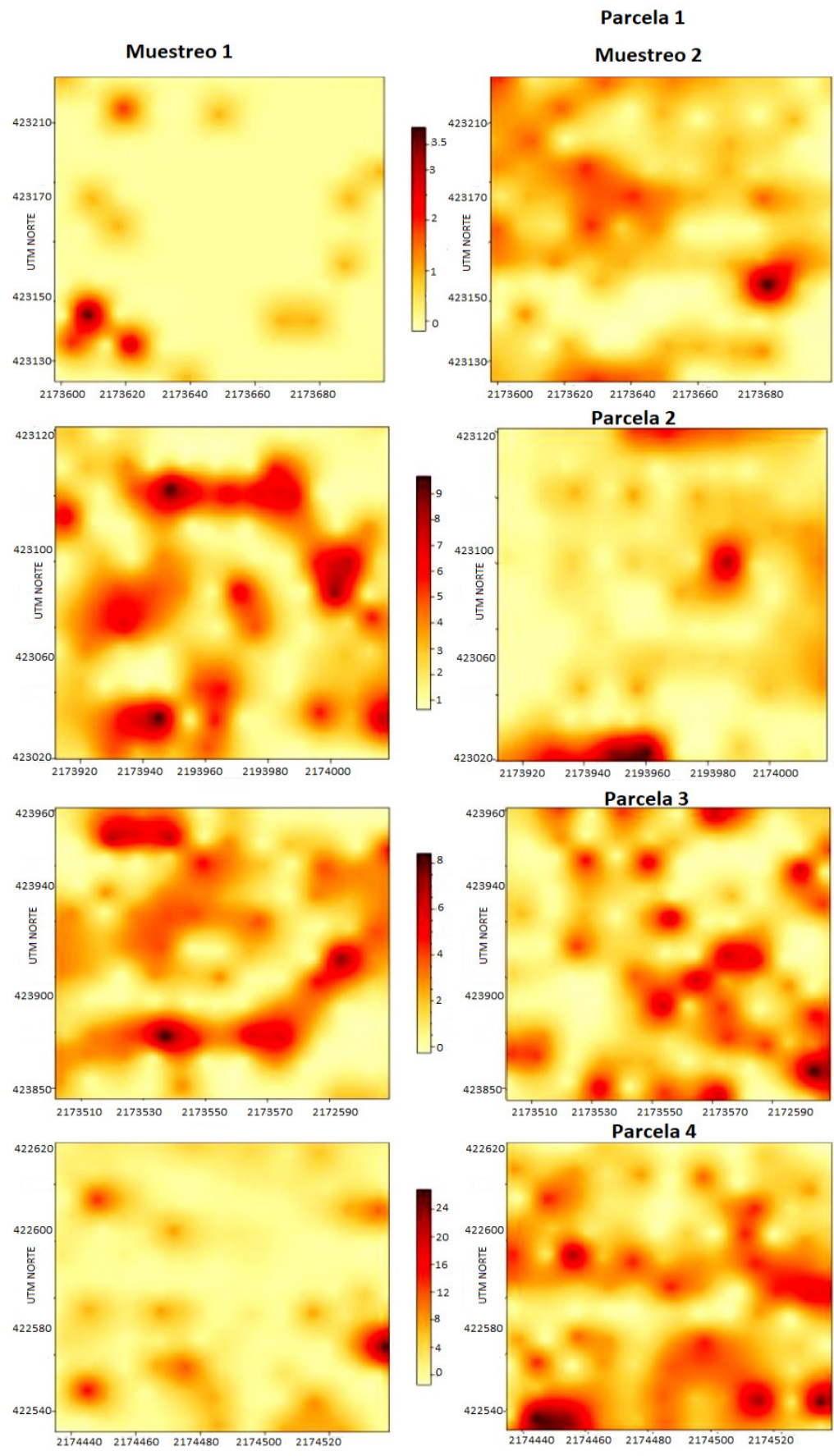
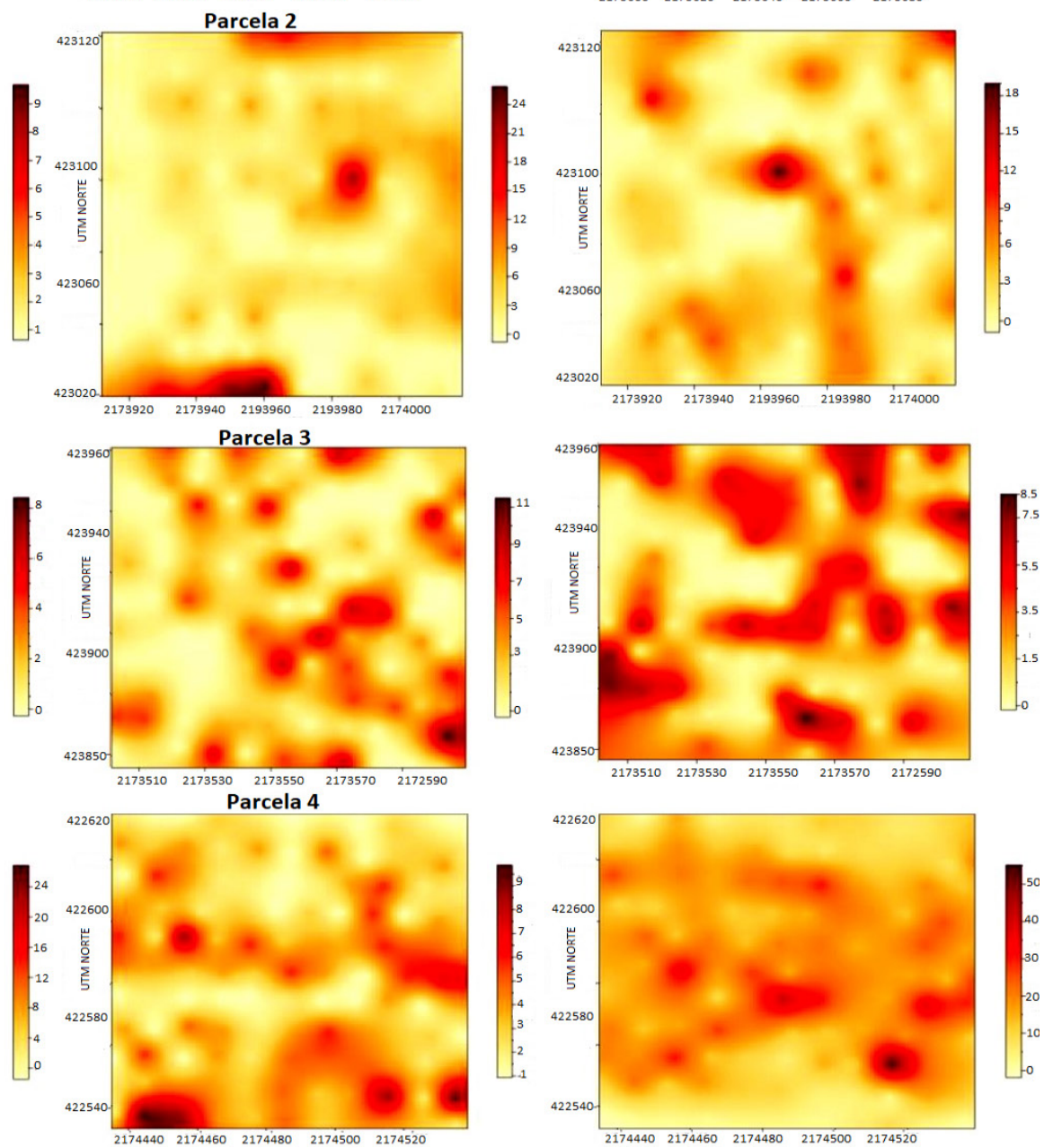
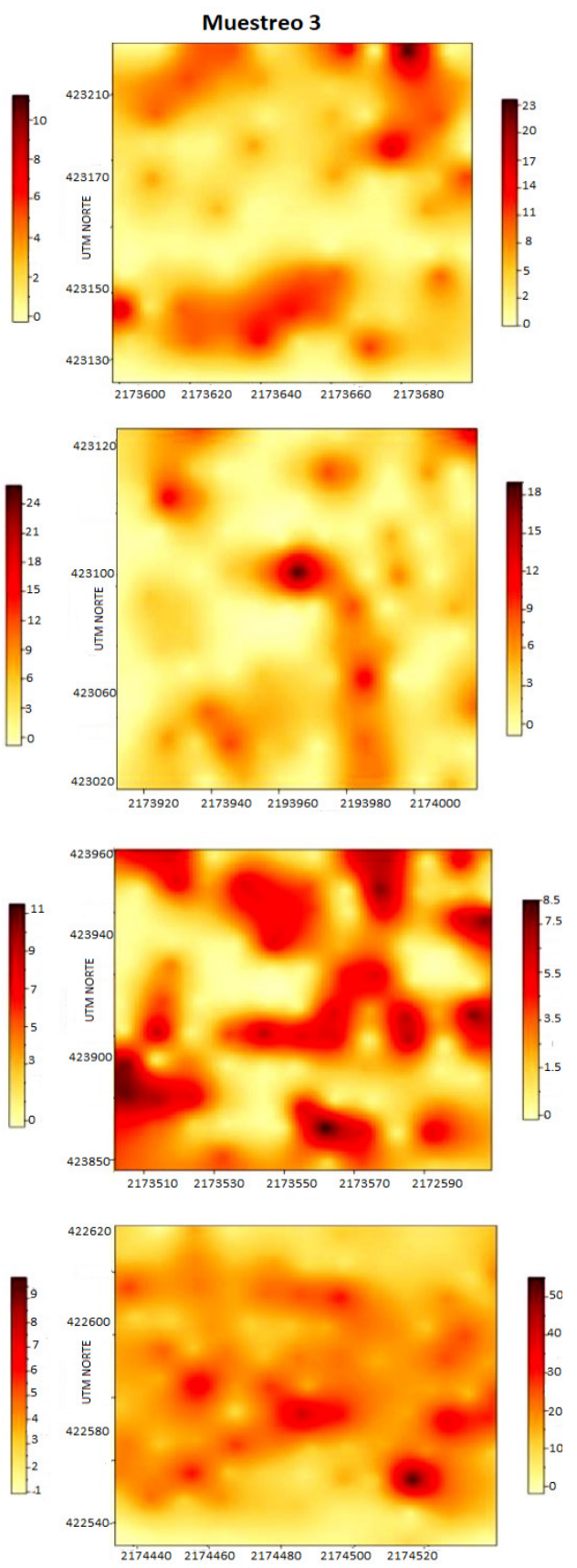

Figura 3. Mapas de densidad de las poblaciones de ninfas de Bactericera cockerelli Sulc., obtenidos en las parcelas del municipio de Jocotitlán Estado de México.

Figure 3. Nymph populations density maps of Bactericera cockerelli Sulc., obtained in the plots of the municipality of Jocotitlán State of Mexico. 
realizados, estos mapas se basan en la técnica de estimación geoestadística conocida como "krigeado", particularmente el ordinario. Contreras et al. (2016) realizaron un estudio de distribución espacial de la enfermedad Candidatus liberibacter solanacearum y de su vector Bactericera cockerelli Sulc en papa, en el cual pudieron obtener mapas de densidad de $B$. cockerelli tanto en huevos, ninfas y adultos, usando el krigeado.

El uso de las técnicas para modelar la distribución espacial de insectos ha mostrado ser una herramienta muy eficiente para determinar la distribución espacial de diversos insectos plaga en cultivos de importancia económica. Diferentes trabajos como el de Jiménez et al. (2000) reportan la distribución temporal y agregada de Thrips palmi Karny en papa en Cuba. Por otra parte, Sciarretta et al. (2008) encontraron una distribución agregada con ajuste al modelo esférico en Lobesia botrana en parcelas de uva. En cuanto a Bressan et al. (2010) determinaron la distribución espacial de Pentastiridius leporinus L., en campos de trigo; Ramírez et al. (2011) reportan la distribución agregada de Bactericera cockerelli en papa; Jiménez et al. (2013) reportan la presencia de poblaciones de trips en tomate de cáscara con distribución agregada con varios centros de agregación visualizados en los mapas obtenidos; sus semivariogramas de la distribución espacial se ajustaron a los modelos esférico y gaussiano; Quiñonez et al. (2015) en todo el ciclo fenológico del cultivo del gladiolo, generando mapas que reflejaron la estructura agregada de la plaga en puntos específicos dentro de la parcela. Por el contrario, Carrillo et al. (2004) realizaron un estudio de la distribución espacial de larvas de escarabaeidos en plantas cultivadas y sus resultados mostraron que las larvas estudiadas no tenían una distribución agregada, esto pudo haber estado relacionado con la amplia polifagia de las larvas.

El comportamiento espacial de las poblaciones de ninfas de $B$. cockerelli de forma agregada en las parcelas de tomate, permite sugerir que se pueden manejar y, por lo tanto, reducir las aplicaciones al dirigir medidas de control hacia los centros de agregación o puntos especíicos en los que se encuentra la plaga, evitando aplicaciones generalizadas o de cobertura total. Todo ello llevará a un ahorro en los insumos, visualizando los focos de infestación a través de los mapas generados. Lo anterior concuerda con lo reportado por Rong et al. (2006) y Esquivel y Jasso-(2014) quienes, respectivamente propusieron el manejo de Locusta migratoria manilensis L., y de Spodoptera exigua Hubner en el cultivo de maíz en puntos específicos de presencia de la plaga.

Los valores de cero encontrados en el efecto pepita en todos los modelos ajustados, permite afirmar que la escala de muestreo utilizada fue la adecuada y el error de muestreo fue mínimo (Oliver y Webster, 1998), esto sugiere que los modelos ajustados tienen un $98 \%$ de confiabilidad. En consecuencia, es válido deducir que más del $90 \%$ de la variación observada en la distribución de las poblaciones de ninfas se logró explicar por la estructura espacial establecida con los semivariogramas (Liebhold y Sharov, 1998). Ramírez et al. (2011) encontraron valores de pepita igual a cero en un estudio de la distribución espacial de Bactericera cockerelli en papa. Valores de cero de la pepita también fueron obtenidos por Solares et al. (2013) en la distribución espacial de trips en plantaciones comerciales de aguacate.

La distribución espacial ajustada al modelo esférico indica que dentro de la parcela analizada existen zonas en que se manifiesta más la plaga respecto al resto de puntos considerados en el muestreo $y$, que se desplaza de un punto específico hacia otros puntos dentro de la parcela, estos puntos de agregación presentan un crecimiento rápido cerca de su origen, pero los incrementos van decreciendo conforme aumenta la distancia (Solares et al. 2013). El ajuste al modelo Gaussiano nos indica que el comportamiento de la agregación de la plaga se expresa de forma continua dentro de la parcela estudiada (Ribes et al., 1998). El ajuste al modelo exponencial indica que la plaga se encuentra ubicada aleatoriamente dentro de la parcela y no sigue un patrón de infestación definido (Ramírez et al., 2013). Los focos de infestación se observan en los mapas obtenidos. Moral (2003) al realizar la distribución espacial de H. armigera y sus variogramas teóricos, encontró que se ajustaron a modelos esféricos o exponenciales, con un efecto pepita. Contreras et al. (2016) encontraron que los semivariogramas para huevos y ninfas de $B$. cockerelli se ajustaron al modelo esférico, lo que indica la agregación de la plaga en ciertas zonas de la parcela.

La distribución de las ninfas de $B$. cockerelli presentó un alto nivel de dependencia espacial en todos los modelos ajustados, esto es indicativo de la existencia de una fuerte relación espacial de la plaga en los puntos muestreados. El alto nivel de dependencia espacial resultó de dividir el efecto pepita entre la meseta que fue menor a $25 \%$ para todos los semivariogramas. Los valores del efecto pepita indicaron una alta dependencia espacial lo cual sugiere que las poblaciones de ninfas dependen entre sí y que el nivel de agregación es alto (Rossi et al., 1992). Maldonado et al. (2017) en un estudio de la distribución espacial de trips en el cultivo de aguacate, utilizando las técnicas de la geoestadística, mostraron la existencia de una estructura espacial agregada, con un nivel de dependencia espacial alto para todos los muestreos realizados.

En los mapas de densidad que se obtuvieron mediante la técnica del krigeado se observan los centros de agregación que presentó la plaga dentro de la parcela, lo cual al momento de proteger el cultivo nos permite dirigir de forma precisa las medidas de control (Acosta et al., 2017; Fleischer et al., 1999; García, 2004; Maldonado et al., 2017; Ribes et al., 1998; Rong et al., 2006; Weisz et al., 1996). Esto ayudará a que los insectos no generen resistencia a los plaguicidas debido a la creación de refugios temporales dinámicos, al no tratar toda la superficie del cultivo (Moral et al., 2011). Con estas acciones se evitará un incremento de la plaga a ciertos niveles poblacionales que puedan causar daños económicos mayores. El comportamiento de los focos de infestación dentro de la parcela permite suponer que $B$. cockerelli se origina de una fuente puntual (Hoddle et al., 2002). 
Los mapas permitieron identificar las áreas de infestación, encontrando que las ninfas no se distribuyeron en el $100 \%$ de la parcela, ubicando áreas en las que se encontraron poblaciones más altas, lo cual concuerda con Quiñonez et al. (2015), quienes encontraron que las poblaciones de trips en el cultivo del gladiolo no infestaron el $100 \%$ de la parcela y estos se encontraban en forma agregada en puntos específicos de infestación. Jiménez et al. (2013) reportaron que las poblaciones de $F$. occidentalis en tomate de cáscara no se distribuyen en la totalidad de las parcelas. Ramírez et al. (2013) visualizaron que los mapas de infestación de $B$. cockerelli Sulc., en papa reflejaron una estructura agregada del insecto el cual no invadió el $100 \%$ de la superficie de la parcela, permitiendo identificar áreas libres de infestación, Ramírez y Porcayo, (2008) reportaron que las ninfas de Jacobiasca lybica Bergenin y Zanon no invadieron el $100 \%$ de las parcelas de vid en estudio, por lo tanto, la infestación no fue uniforme. Por el contrario, Jiménez et al. (2008), quienes determinaron la distribución espacial y mapeo de Curculio elephas Gyllenhal en Quercus ilex L, elaboraron cuatro mapas de densidad, uno por cada año, utilizando el método de la distancia inversa como interpolador del programa Surfer 7; estos mapas indican que el insecto se localizó por toda la parcela con una distribución espacial agregada.

\section{CONCLUSIONES}

La distribución espacial de las poblaciones de ninfas de $B$. cockerelli se presenta en agregados en las parcelas de estudio, dicha agregación se visualiza perfectamente en los mapas de densidad elaborados. La infestación no se presenta en el $100 \%$ de las parcelas, lo cual resulta de relevancia ya que es posible focalizar las medidas de acción sobre las áreas específicas de infestación. Un uso más eficiente de la geoestadística para el manejo de las poblaciones de $B$. cockerelli se vería beneficiado sí se complementa con el conocimiento del umbral económico del psílido en las zonas de estudio, y así solo establecer medidas de control cuando se rebase dicho umbral.

\section{REFERENCIAS}

Acosta, G.A.D., Ramírez, D.J.F., Rivera, M.R., Figueroa, D.K., Lara, A.V., Maldonado, F.I., y Tapia, R.A. 2017. Distribución espacial de Trips spp. (Thysanoptera) y evaluación de su control mediante el depredador Amblyseius swirskii en el cultivo de aguacate en México. Southwestern Entomologist, 42(2), $435-446$.

Bressan, A., Moral García, F.J., Sémétey, O., y Boudon-Padieu, E. 2010. Spatio-temporal pattern of Pentastiridius leporinus migration in an ephemeral cropping system. Agricultural and Forest Entomology, 12(1), 59 - 68.

Cambardella, C., Moorman, T., Novak, J., Parkin, T., Karlen, D., Turco, R., Konopka, A. 1994. Field scale variability of soil properties in central Iwa soils. Soil. Sci. Soc. Am. J. 58: 1501 $-1511$.

Carrillo, R., Pape, H., Neira, M., Balocchi, O. 2004. Distribución espacial de larvas de dos especies de escarabeidos nativos en respuesta a plantas cultivadas. Sociedad Chilena de Entomología, Santiago (Chile).
Contreras, R.A., Gutiérrez, I.A.T., Silva, R.H.V., Sánchez, P.J.R., Laguna, C.A., Ramírez, D.J.F. 2016. Distribución espacial de Candidatus Liberibacter solanacearum y Bactericera cockerelli (Sulc) (Hemiptera: Triozidae) en papa (Solanum tuberosum L.). Southwestern Entomologist 41 (1): 105 - 114.

Englud, E., Sparks, A. 1988. GEO-EAS (Geostatistical Environmental Assessment Software) User's Guide. U.S. Environmental protection agency document EPA/600/488/033. Environmental Monitoring Systems Laboratory, Las Vegas, NV, USA.

Esquivel, H.V., Jasso, G.Y. 2014. Distribución espacial y mapeo de gusano soldado en seis localidades del Estado de México, en el año 2011. Revista Mexicana de Ciencias Agrícolas. 5: $923-935$.

Fleischer, S.J., Blom, P E., Weisz, R. 1999. Sampling in precision IPM: When the objective is a map. The American Phytopathological Society 89 (11): 115 - 118.

García, F.M. 2004. Aplicación de la geoestadística en las ciencias ambientales. Revista Ecosistemas 13 (1). 78 - 86.

Hoddle, M.S. 2002. Developmental and reproductive biology of Scirtothrips perseae (Thysanoptera: Thripidae): a new avocado pest in California. Bulletin of Entomological Research 92 (04): $279-285$.

Isaaks, E., Srivastava, M. 1989. Spatial continuity measures for probabilistic and deterministic geostatistics. Mathematical Geology. 20 (4): 313 - 341.

Jiménez, J., Cortiñas, J., López, D. 2000. Distribución temporal y espacial y consideraciones para el monitoreo de Thrips palmi en papa en Cuba. Manejo Integrado de Plagas (CATIE). Set 2000. 57: $54-57$.

Jiménez, A., López, M., González, A. J., Ocete, M., Soria, F. 2008. Distribución espacial y mapeo de Curculio elephas Gyllenhal en encina (Quercus ilex L.). Agrociencia. 12: 35 - 43.

Jiménez, R.D.L.A., Ramírez, D.J.F., Sánchez, P.J.R., Salgado, S.M.L., Laguna, C.A. 2013. Modelización espacial de Frankliniella occidentalis (Thysanoptera: Thripidae) en tomate de cáscara por medio de técnicas geoestadísticas. Revista Colombiana de Entomología. 39 (2): 183 - 192.

Journel, A., Huijbregts, C. J. 1978. Mining geostatistics. Academic Press, London, Reino Unido. 600 p.

Liebhold, A.M., Sharov, A. 1998. Testing for correlation in the presence of spatial autocorrelation in insect count data. pp. 111-117. In: Population and community Ecology for insect management and conservation. CRC Press. ISBN: 905410930 0 . Rotterdam, Netherlands.

Liefting, L.W., Perez-Egusquiza, Z.C., Clover, G.R.G., Anderson, J.A.D. 2008. A new 'Candidatus Liberibacter'species in Solanum tuberosum in New Zealand. Plant Disease, 92(10), $1474-1474$.

Maldonado-Zamora, F.I., Ramírez-Dávila, J.F., Lara-Diaz, A.V., Rivera-Martinez, R., Acosta-Guadarrama, A.D., FigueroaFigueroa, D.K., y Tapia-Rodríguez, A. 2017. Estabilidad Espacial y Temporal de la Distribución de Trips 1 en el Cultivo de Aguacate en el Estado de México. Southwestern Entomologist, 42(2), $447-462$.

Moral-García, F.J. 2003. Analysis of the spatio-temporal distribution of Helicoverpa armígera $\mathrm{Hb}$. in a tomato field using a stochastic approach. Biosystems Engineering 93 (3): $253-259$.

Moral, F.J., Terron, J.M., Rebollo, F.J. 2011. Site-specific management zones based on the Rasch model and geostatistical techniques. Comp. Electron. Agri. 75: 223 - 230. 
Oliver, M., Webster, R. 1998. How geostatistics can help you. Soil Use and Management 7 (4): 206 - 217.

Pérez, M., Granados, A. 2001. Nitro-phosphoric fertilization in cascara tomato Physalis ixocarpa Brot., Irrigation in Irapuato, Gto, Mexico. University certificate. 11(001): 19 - 25.

Quiñones, V.R., Sánchez, P.J.R., Pedraza, E.A.K., Castañeda, V.A., Gutiérrez, I.A.T., Ramírez, D.J.F. 2015. Análisis Espacial de Thrips spp. (Thysanoptera) en el Cultivo de Gladiolo en la Región Sureste del Estado de México, México. Southwestern Entomologist. 40 (2): 397 - 408.

Ramírez, D.J.F., Porcayo, C.E. 2008. Distribución espacial de las ninfas de Jacobiasca lybica (Hemiptera: Cicadellidae) en un viñedo en Andalucía, España. Revista Colombiana de Entomología. 34 (2): 169 - 175.

Ramírez, D.J.F., Porcayo, C.E., Sánchez, P.J.R. 2011. Análisis de la distribución espacial de Bactericera cockerelli Sulc (hemiptera: triozidae) en Solanum tuberosum L. en Donato Guerra, México. Boletín del Museo de Entomología de la Universidad del Valle. 12 (1): 12 - 24.

Ramírez, D.J.F., Solares, A.V.M., Figueroa, F.D.K., Sánchez, P.J.R. 2013. Comportamiento espacial de trips (Insecta: Thysanoptera), en plantaciones comerciales de aguacate (Persea americana Mill.) en Zitácuaro, Michoacán, México. Acta Zoológica Mexicana. 29 (3): 545 - 562.

Ribes, D.M., Bascuñana, C.M., Avilla, H.J. 1998. Estudio de la distribución espacial de Cydia pomonella (L.) y Pandemis heparana (Denis \& Schiffermüller) en Torregrossa (Lleida) mediante métodos geoestadísticos. Boletín de Sanidad Vegetal Plagas 24 (4): 935 - 948.
Rong, J., Dian, M.L., Bao, Y., Zhe, X., Dong, L. 2006. Spatial Distribution of Oriental Migratory Locust (Orthoptera: Acrididae) Egg Pod Populations: Implications for Sitespecific Pest Management. Environmental Entomology. 35: $1244-1248$.

Rossi, R., Mulla, J., Journel, G., Franz, H. 1992. Geostatical tools for modeling and interpreting ecological spatial dependence. Ecological Monographs. 62 (2): 277 - 314.

Samper, F.J., Carrera, J. 1996. Geoestadística, aplicaciones a la hidrogeología. Centro Internacional de Métodos Numéricos en Ingeniería, España.

Sciarretta, A., Zinni, A., Mazzocchetti, A., Trematerra, P. 2008. Spatial Analysis of Lobesia botrana (Lepidoptera: Tortricidae) Male Population in a Mediterranean Agricultural Landscape in Central Italy. Environmental Entomology April 2008. 37 (2): $382-390$.

SIAP. (2016). Servicio de Información Agroalimentaria y Pesquera. SIAP. http://www.siap.gob.mx/ (Consulta: 7 de noviembre 2014).

Solares, V.M., Ramirez, D.J.F., Sanchez, P.J.R. 2013. Spatial distribution of thrips (Insecta: Thysanoptera) in avocado cultivation (Persea americana Mill). Bulletin of the Museum of Entomology of the Universidad del Valle. 12 (2): 1 - 12.

Surfer, V. 1993. Version 5.01, Surface Mapping System, Golden Software. Inc., Golden, Colorado, USA.

Weisz, R., Fleischer, J., Smilowitz, Z. 1996. Site specific integrated pest management for high value crops: Impact on potato pest management. Journal of Economic Entomology 89: 501 -509 . 